\title{
Statistics and Thermodynamics of Fe-Cu Alloys at High Temperatures.
}

\author{
Leonid Son ${ }^{1, *}$, Valery Sidorov ${ }^{1,2}$, and Nikolay Katkov ${ }^{1,2}$ \\ ${ }^{1}$ Ural Federal University, 19 Myra st., Ekaterinburg, Russia \\ ${ }^{2}$ Ural State Pedagogical University, 26 Cosmonavtov ave., Ekaterinburg, Russia
}

\begin{abstract}
Statistics and thermodynamics of binary $\mathrm{Fe}-\mathrm{Cu}$ system is described in terms of local state representation combined with the account of nonergodic microheterogeneity in terms of frozen fluctuations of chemical potential. It is shown that the microheterogeneity in this system is an equilibrium property up to $1700^{\circ} \mathrm{C}$.
\end{abstract}

\section{Introduction}

$\mathrm{Fe}-\mathrm{Cu}$ system is a binary system with limited miscibility of the components. Most of such systems correspond to the eutectic or monotectic type of the concentration - temperature phase diagram, but $\mathrm{Fe}-\mathrm{Cu}$ system demonstrates peritectic triple point [1] and strong microheterogeneity in the liquid state [2].

In [3], we suggested the description of binary systems with limited miscibility, which is based on the local state representation of condensed matter statistics and thermodynamics. The description arises from the melting theory [4] based on the Potts model [5].

In the theory, it is suggested that small local volume (first coordination shell) of condensed matter may be characterized by strict local order with local anisotropy. The interaction between neighboring local volumes makes the coinciding mutual orientation of the local anisotropy axis preferable, i.e. corresponding to the energy minima. The disorientation leads to the energy rising. Thus, one can divide all possible orientations into $n$ orientation shells, and suppose that the energy of interaction of nearest neighbors is $-J$, if their orientations get into one and the same shell, and 0 otherwise. The parameters $J, n$ characterize the depth and the width of the orientation interaction, respectively. The system is obviously being described by the $n$ - states Potts model, which demonstrates first order phase transition from the orientationally ordered state at low temperatures (all local volumes get into one and the same orientation shell) to the disordered one (all orientations have equal probabilities) at high temperatures. It is natural to interpret the transition as a melting. In [3], we applied this approach to binary systems and show that systems with limited miscibility may be successfully described.

In [6], we introduced a way to account nonergodic microheterogeneity in binary systems, which arises from the dynamical limitations imposed on particles diffusion. It was accounted by frozen fluctuations of chemical potential. Their mean square value serves as

${ }^{*}$ Corresponding author: $\underline{1 d s o n @ y \text { yandex.ru }}$ 
an additional thermodynamic parameter. As a result, one gets a possibility to calculate boundaries of microheterogeneity at the phase diagram.

In the present work, we combine the approaches $[3,6]$ to describe the $\mathrm{Fe}-\mathrm{Cu}$ system.

\section{The model}

\subsection{Local state modeling}

The state of small volume in binary system located at point $r$ may be described by $2-$ index local state vector $\sigma_{k}^{i}(r)$, where the upper index $i=\overline{1,2}$ corresponds to the component, and the lower numerates the orientation. Here, the overbar denotes the limits of the index. In general case, $k=\overline{1, n}$ when $i=1$, and $k=\overline{1, m}$ when $i=2$. Thus, the numbers $n, m$ characterize the width of orientation interaction for the first and second components respectively. The vector is defined by the rule

$$
\sigma_{k}^{i}(r)=\left\{\begin{array}{c}
1, \text { if } r \text { is occupied by component } i \text { in } k^{\text {th }} \text { orientation } \\
0, \text { otherwise }
\end{array}\right.
$$

The Hamiltonian of system in terms of these variables is

$$
\begin{aligned}
H\{\sigma\} & =\frac{1}{2} \sum_{r, r^{\prime}} \sigma_{k}^{i}(r) M_{k l}^{i j}\left(r-r^{\prime}\right) \sigma_{l}^{j}\left(r^{\prime}\right)+\sum_{r} \mu_{k}^{i}(r) \sigma_{k}^{i}(r), \\
\mu_{k}^{i} & =\left\{\begin{array}{l}
\mu, i=1 \\
0, i=2
\end{array}\right.
\end{aligned}
$$

Here, $\mu$ is the chemical potential, and kernel $M_{k l}^{i j}\left(r-r^{\prime}\right)$ differs from zero only for nearest neighbors and realizes the idea of orientation interaction

$$
M_{k l}^{11}=-J_{1} \delta_{k l}, \quad M_{k l}^{22}=-J_{2} \delta_{k l} .
$$

In [3], inter - component terms of the kernel were taken in the form

$$
M_{k l}^{12}=M_{k l}^{21}=\varepsilon, \forall k, l
$$

This form is appropriate, if the two components differ sharply from each other (have different local structures or interatomic distances) - then one can suppose that if two different local structures share a boundary, then the energy of their interaction is $\varepsilon$ regardless of their orientation. For the $\mathrm{Fe}-\mathrm{Cu}$ system, the interaction kernel should be modified. First of all, the two metals have similar local structures (corresponding to FCC close packed lattice), so that we keep the form of eq.(3) and choose $m=n$. Besides, if the two local structures are similar to each other, then coinciding orientations are preferable for nearest neighbors in the case of different components also, and instead of (4) one writes 


$$
M_{k l}^{12}=M_{k l}^{21}=\varepsilon+J_{0} \delta_{k l}
$$

This form of interaction contains itercomponent tension $\varepsilon$ and energy benefit $J_{0}$ for coinciding orientations. The next step is to calculate the partition and thermodynamic potential

$$
Z=\sum_{\{\sigma\}} \exp \left[-\frac{H\{\sigma\}}{T}\right], \quad F=-T \ln Z
$$

This can be made in the mean - field approximation, which implies introduction of the mean value

$$
<\sigma_{j}^{i}(r)>=\omega_{j}^{i}
$$

in terms of which the unequilibrium thermodynamic potential should be written out. As a result, one gets the Landau theory, where (7) plays the role of order parameter. In detail, the procedure is presented in [3]. The result is as follows. The nonequilibrium Landau potential is given by

$$
\begin{aligned}
& F=\frac{1}{2} \omega_{k}^{i} M_{k l}^{i j} \omega_{l}^{j}-T \ln Z_{M F}, \\
& Z_{M F}=\sum_{i, k} \exp \left(\frac{1}{T} M_{k l}^{i j} \omega_{l}^{j}+\frac{1}{T} \mu_{k}^{i}\right) .
\end{aligned}
$$

The structure of the order parameter (7) is

$$
\begin{aligned}
& \omega_{1}^{1}=x \sigma_{1}, \quad \omega_{k \neq 1}^{1}=x \frac{1-\sigma_{1}}{n-1}, \\
& \omega_{1}^{2}=(1-x) \sigma_{2}, \quad \omega_{k \neq 1}^{2}=(1-x) \frac{1-\sigma_{2}}{n-1}
\end{aligned}
$$

Here, $x$ is the first component concentration. We assigned number 1 to the orientation that is preferable in the crystal state, so that $\sigma_{1}, \sigma_{2}$ are the parameters of crystal order (for the first and second components respectively): in the crystal state, these values are close to unity, while in the liquid $\sigma_{1,2}=\frac{1}{n}$. In terms of $(3-5,9)$, quadratic and logarithmic parts of this expression take the form 


$$
\begin{aligned}
F_{\text {quad }} & =\frac{J_{1}}{2} x^{2} \sigma_{1}^{2}+\frac{J_{1}}{2} x^{2} \frac{\left(1-\sigma_{1}\right)^{2}}{n-1}+ \\
& +\frac{J_{2}}{2}(1-x)^{2} \sigma_{2}^{2}+\frac{J_{2}}{2}(1-x)^{2} \frac{\left(1-\sigma_{2}\right)^{2}}{n-1}+ \\
& +J_{0} x(1-x)\left(\sigma_{1} \sigma_{2}+\frac{\left(1-\sigma_{1}\right)\left(1-\sigma_{2}\right)}{n-1}\right)+\varepsilon x(1-x), \\
Z_{M F} & =E_{1}^{(1)}+(n-1) E_{2}^{(1)}+E_{1}^{(2)}+(n-1) E_{2}^{(2)}
\end{aligned}
$$

where

$$
\begin{aligned}
& E_{1}^{(1)}=\exp \left[\frac{J_{1}}{T} x \sigma_{1}+\frac{J_{0}}{T}(1-x) \sigma_{2}+\frac{\varepsilon}{T}(1-x)+\frac{\mu}{T}\right] \\
& E_{2}^{(1)}=\exp \left[\frac{J_{1}}{T} x \frac{1-\sigma_{1}}{n-1}+\frac{J_{0}}{T}(1-x) \frac{1-\sigma_{2}}{n-1}+\frac{\varepsilon}{T}(1-x)+\frac{\mu}{T}\right] \\
& E_{1}^{(2)}=\exp \left[\frac{J_{2}}{T}(1-x) \sigma_{2}+\frac{J_{0}}{T} x \sigma_{1}+\frac{\varepsilon}{T} x\right] \\
& E_{2}^{(2)}=\exp \left[\frac{J_{2}}{T}(1-x) \frac{1-\sigma_{2}}{n-1}+\frac{J_{0}}{T} x \frac{1-\sigma_{1}}{n-1}+\frac{\varepsilon}{T} x\right]
\end{aligned}
$$

Minimizing $F$ with respect to $\omega_{k}^{i}$ at fixed temperature $T$, one gets mean field equations on the crystal order parameters

$$
\sigma_{1}=\frac{E_{1}^{(1)}}{E_{1}^{(1)}+(n-1) E_{2}^{(1)}}, \quad \sigma_{2}=\frac{E_{1}^{(2)}}{E_{1}^{(2)}+(n-1) E_{2}^{(2)}}
$$

and equation on the phase separation

$$
x=\frac{E_{1}^{(1)}+(n-1) E_{2}^{(1)}}{Z_{M F}} .
$$

The scheme of the phase diagram calculation is as follows. First, at fixed temperature $T$ and concentration $x$ one has to solve equations (12). Each of these equations may have two stable solutions $\sigma_{1,2} \approx 1$, and $\sigma_{1,2}=\frac{1}{n}$. The first corresponds to the crystal, and second - to the liquid.

Then, for all solutions of (12), which represent possible phases, the thermodynamic potential (8) has to be plotted as a function of $(x, T)$, afterwards the phase diagram may calculated by standard shared tangent method. Equation (13) serves to indicate phase separation in liquid: when this equation has single solution at all values of chemical 
potential $\mu$, then the phase separation is impossible, and it takes place if multiple solutions realize while changing $\mu$

\subsection{Account of nonergodic diffusivity}

In [6], it was shown that nonergodic diffusivity may be accounted in terms of random frozen spatial fluctuations of chemical potential. The corresponding corrections may be derived by substituting

$$
\frac{\mu}{T} \Rightarrow \frac{\mu}{T}+\frac{\Delta \mu}{T}
$$

where $\Delta \mu$ is random spatial fluctuation. Then one averages $(10,12,13)$ over these fluctuations. Since equations (12) contain no $\mu$, they do not experience any changes. Correction to the thermodynamic potential is

$$
\begin{aligned}
F_{\text {corr }} & =F+\frac{\partial^{2} F}{\partial(\mu / T)^{2}} C=F_{\text {quad }}-T \ln Z_{M F}- \\
& -T C\left(\frac{E_{1}^{(1)}+(n-1) E_{2}^{(1)}}{Z_{M F}}-\frac{\left(E_{1}^{(1)}+E_{2}^{(1)}\right)^{2}}{Z_{M F}^{2}}\right)
\end{aligned}
$$

where the dimensionless constant $\mathrm{C}$ is the mean square of $\Delta \mu / T$ fluctuations and should be treated as an additional phenomenological parameter. Corrected form of eq.(13) is

$$
\begin{aligned}
x & =\frac{E_{1}^{(1)}+(n-1) E_{2}^{(1)}}{Z_{M F}}+C \frac{E_{1}^{(1)}+(n-1) E_{2}^{(1)}}{Z_{M F}^{3}} \times \\
& \times\left(1-3 \frac{E_{1}^{(1)}+(n-1) E_{2}^{(1)}}{Z_{M F}}+2 \frac{\left(E_{1}^{(1)}+(n-1) E_{2}^{(1)}\right)^{2}}{Z_{M F}^{2}}\right)
\end{aligned}
$$

In the next section we calculate the $\mathrm{Fe}-\mathrm{Cu}$ phase diagram using $(12,15,16)$.

\section{Phase diagram calculation.}

We start with determination of model parameters, which allow one to fit the experimental diagram presented in fig.1. We did not take into account the phase transformation in iron near melting point, and treated iron having FCC local order all over the diagram. Also, we do not consider the low - temperature $\gamma \leftrightarrow \beta$ transformation.

First, for the close - packed systems, number of orientation shells $n=12$ is appropriate [4]. Parameters $J_{1}, J_{2}$ were chosen to reproduce melting temperatures of the pure components:

$$
J=T_{\text {melt }} \frac{2(n-1)}{(n-2)} \ln (n-1) .
$$




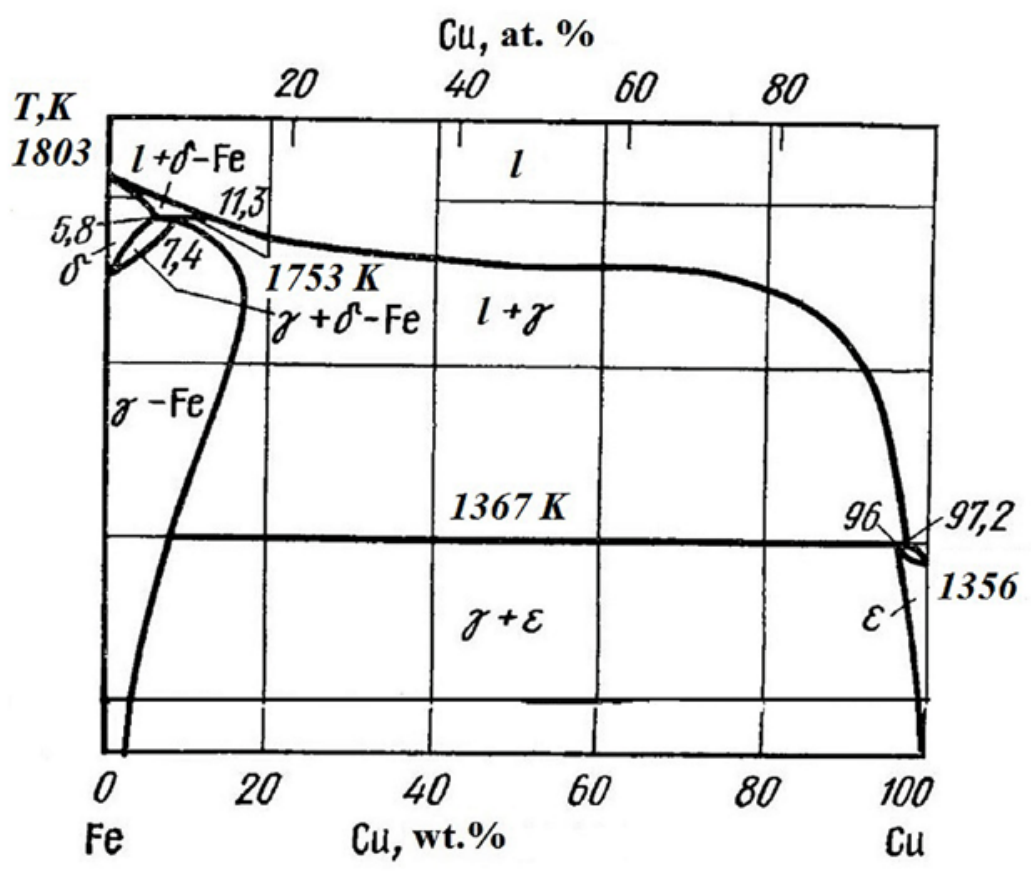

Fig. 1. Experimental $\mathrm{Fe}-\mathrm{Cu}$ phase diagram according to [1].

We used energy scale in Kelvin degrees, so that $J_{1}=9443$ and $J_{2}=7153$. Parameter $J_{0}$ was chosen to reproduce the slope of liquidus lines near pure components, $J_{0}=8400$. Parameters

$\varepsilon, C$ were determined from peritectic temperature and critical point in the undercooled illiquid. The later is $T_{c}=1670^{\circ} \mathrm{K}[8]$. We used the relation

$$
T_{c}=\left(1-\frac{C}{2}\right)\left(\frac{J_{1}+J_{2}-2 J_{0}}{4 n}-\varepsilon\right),
$$

which may be derived from (16). We varied $\varepsilon$ to reproduce the peritectic triple point, and got $\varepsilon=-4010, C=0.32$.

The results of calculation are presented in Fig.2. Equilibrium diagram is drawn by solid lines. One can see that the diagram is reproduced rather well. The lower dashed line corresponds to the phase separation [8] in undercooled liquid. The upper dashed curve eliminates area, where nonergodic microheterogeneity [6] takes place (shadowed area in fig.2). It was calculated as a miscibility cupola according to eq. (13). Nonergodic microheterogeneity in $\mathrm{Fe}-\mathrm{Cu}$ liquid system was discovered in [2] by viscosity measurements, and justified in [8]. Note, that dashed lines do not correspond to any line of equilibrium phase transition. Thus, we can to fit the phase diagram by 6 - parameter model, including melting and phase separation in undercooled liquid, and to explain the features of system behavior in the liquid state. Thus, the thermodynamics of melting and dissolution in the $\mathrm{Fe}-\mathrm{Cu}$ system is described rather well. 


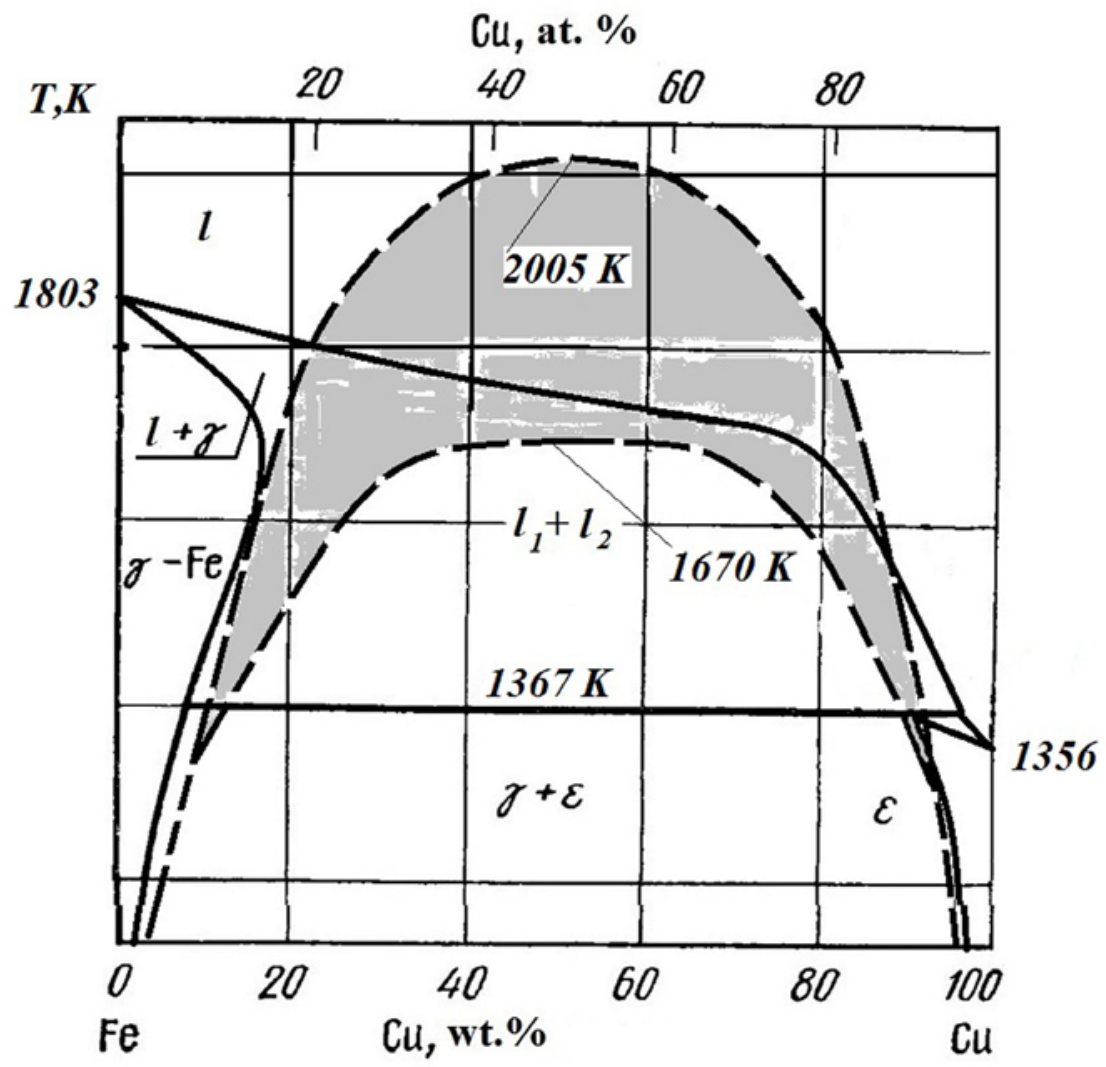

Fig.2. Phase diagram, calculated in the present paper.

The work was supported by Russian Ministry of Science and Education (Government job 2014/392 projects 2391 and 1177), and by Government of the Russian Federation, act 211 contract № 02.A03.21.0006. L.Son is also grateful to RFBR (grant 14-02-00359)

\section{References}

1. Lyakishev N.P., Bannykh O.A., Rokhlin L.L., et. al. Phase Diagrams of Binary Metallic Systems. Handbook. Moscow, Mashinostrojenie, 1996 (in Russian)A.

2. V. S. Guschin, D. B. Shulgin, B. A. Baum, and T.P.Buhler, Metallofizika 11 (6), 33 (1989)

3. Son L., Patashinski A., Rusakov G., Ratner M., Physica A, 248 (3-4), 305-322 (1998)

4. A.C.Mitus, A.Z.Patashinski, Sov.Phys. JETP, 53, 798 (1981); Phys. Lett. 87A, 179 (1982)

5. F.Y.Wu. Rev.Mod.Phys, 54, 239 (1982).

6. L.D.Son .Physica A, 449C, 395-400 (2016)

7. Turchanin M.A., Agraval P.G., Nikolaenko I.V. J. Phase Equilibria, 24, (4) 307-319 (2003)

8. O. A. Chikova, M. A. Vityunin, V. P. Chentsov, and G.V. Sakun, Colloidnyi Zhurnal 72 (2), 1-7 (2010) 